The Chittagong Univ. J. Sci. 42(1): 1-23, 2020

\title{
Participatory Tree Carbon Measurement in Komolchari Village Common Forests in Chittagong Hill Tracts
}

\author{
Md. Danesh Miah*, Md. Arif Chowdhury and Mohammed Jashimuddin \\ Institute of Forestry and Environmental Sciences, University of Chittagong, Chittagong 4331, \\ Bangladesh \\ *Corresponding author; E-mail: dansmiah@gmail.com, danesh@cu.ac.bd
}

Manuscript received on 05 May, 2019, Revised manuscript received on 15 March, 2020 and accepted on 01 November, 2020.

\begin{abstract}
Climate change is taking place at a horrifying rate due to the increasing concentration of $\mathrm{CO}_{2}$ in the atmosphere. REDD+ has been considered as a low-cost approach to reducing atmospheric carbon. A study on measurements of tree carbon through a participatory approach was conducted in Komolchari Village Common Forest (VCF) of Khagrachari under the Chittagong Hill Tracts to examine the contribution of local participants in the measurement of tree carbon. From the study, it was estimated that total tree biomass density measured by the forestry experts and the local participants were $147.40 \pm 31.26$ tha ${ }^{-1}$ and $135.95 \pm 27.54$ tha $^{-1}$, respectively, where total carbon density for trees was $73.70 \pm 15.63$ tha ${ }^{1}$ and $67.98 \pm 13.77$ tha $^{-1}$, respectively. Furthermore, in the case of saplings, the estimated total biomass density measured by the forestry experts and the local participants were $33.63 \pm 3.50$ tha ${ }^{-1}$ and $32.41 \pm 3.09$ tha $^{-1}$, respectively, where estimated total carbon density for saplings was $16.82 \pm 1.75$ tha $^{-1}$ and $16.21 \pm 1.55$ tha $^{-1}$, respectively. From all of the findings, it was observed that a participatory approach was successfully conducted in the study area to collect data on the measurement of tree carbon. The study will help bring the profit in the carbon trade by reducing transaction costs in the case of collecting data on tree carbon measurement. The findings of the study can be useful for REDD+ implementation in Bangladesh.
\end{abstract}

Keywords: Biomass; Trees; Sapling; Aboveground carbon; Belowground carbon DOI: https://doi.org/10.3329/cujsv42i1.54235

বায়ুমন্ডলে বৃদ্ধিপ্রাপ্ত কার্বন ডাইঅক্সাইড এর কারনে জলবায়ু পরিবর্তন দ্রংতগতিতে হচ্ছে। বায়ুমন্ডলের কার্বন কমানোর জন্য রেড+ একটি কম খরচের ব্যবস্থা হিসেবে পরিগণিত হয়েছে। গাছের কার্বন পরিমাপের ক্ষেত্রে স্থানীয় অংশগ্রহণকারীদের অবদান জানার জন্য পার্বত্য চট্টগ্রামের খাগড়াছড়ি জেলার কমলছড়ি মৌজাবনে অংশগ্রহণমূলক 
2 Danesh Miah, Md. Arif Chowdhury and Mohammed Jashimuddin

ব্যবস্থার মাধ্যমে গাছের কার্বন পরিমাপের একটি গবেষণা পরিচালনা করা হয়। গবেষণায় দেখা গেছে, বনবিদ্যায়

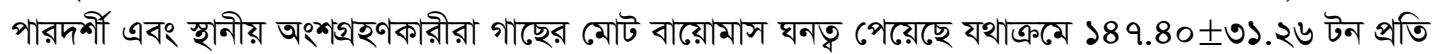

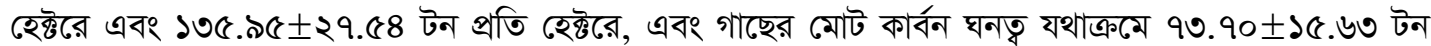

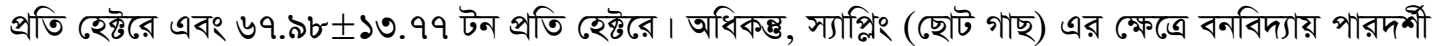

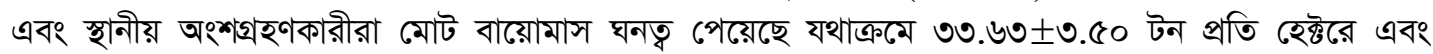

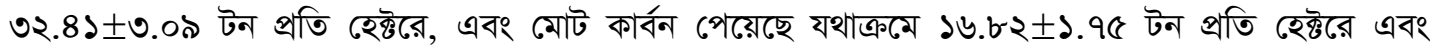

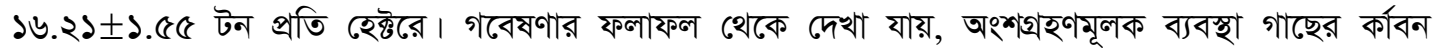
পরিমাপের ডাটা সংগ্রহের ব্যাপারে সফলভাবে কাজ করেছে। এই গবেষণা ট্রানজেকশান-খরচ কমিয়ে কার্বন বাণিজ্যকে লাভজনক করার ব্যাপারে সহযোগীতা করবে। এই গবেষণার ফলাফল বাংলাদেশে রেড+ কার্যক্রম বাস্তবায়নের ক্ষেত্রে প্রয়োজনীয় হবে।

\section{Introduction}

Climate change, including negative impacts globally, is an alarming issue nowadays. Global carbon emission is increasing day by day where deforestation and forest degradation are the significant causes of this. An approach to reducing the impact of climate change providing financial support to developing countries for reducing forest land degradation, deforestation and emission of greenhouse gas through the medium of carbon credits or maintaining markets for carbon has been provided by the "Reducing Emissions from Deforestation and forest Degradation and supporting the role of conservation, sustainable management of forests and enhancement of forest carbon stocks (REDD+)" in developing countries [1].

REDD+ program provides scope to create markets for carbon through sustainable forest management, which has essential potential in reducing greenhouse gas emissions by promoting forest management, protecting forest stocks and preventing forest fires [2]. In Tanzania, three REDD+ pilot programs were adopted to assess the data by estimating various types of costs such as opportunity, implementation, transaction costs of REDD+ to improve applicable framework to estimate the cost elements of REDD+ [3]. Monitoring activities of biodiversity research organizations and REDD+ together improve the monitoring capabilities, evaluation of information in a national sphere, and enhance the ecological and socio-economic conditions of 
REDD+ [4]. In Nepal in 2009, a pilot project of REDD+ found that REDD+ project can manage the sustainable forest management and take part in carbon sequestration matter without imposing any effects on local livelihoods [2]. To run a verifiable carbon benefit, a framework of REDD+ project should be implemented containing a primary carbon inventory, a baseline and reference level, carbon monitoring in a continuous process and evaluation program [5]. From nine pilot projects of REDD+ in Kigoma, Western Tanzania provided ahelping hand to a community for reducing deforestation and degradation of forest, emission of carbon, protect and improve biodiversity and other services of an ecosystem [6]. In Masindi district of Western Uganda in 2009, a REDD+ pilot project was conducted to find out a baseline including the socioeconomic conditions of households by comparing with follow-up surveys which helped to identify the potentiality of REDD+ pilot project on poverty reduction and sustainable development while the survey was done in several villages surrounded by communally owned forest and Ongo community forest [7]. It is an important topic to estimate carbon following a standard cost-effective way. Furthermore, in 2011, a project was done under REDD+ based on a community of Nepal for Nepal's Community Forest Management by Asia Network for Sustainable Agriculture and Bioresources (ANSAB), International Centre for Integrated Mountain Development (ICIMOD) and the Federation of Community Forest Users Nepal (FECOFUN) [8]. REDD+ is now in the concentration of developing countries for providing a source of international funding and at now, at least 65 countries are eager to accept the REDD+ [9].

In terrestrial vegetation, forests play as a significant reservoir of carbon, containing $80 \%$ of all carbon stored, but when forest lands are degraded, the stored carbon is released to the atmosphere [10]. Furthermore, in the case of regulating the atmospheric concentration of $\mathrm{CO}_{2}$, diverse, and vital roles are played by forests [11]. In the case of storing atmospheric carbon, trees play an essential role while storing rate depends on the age and growth rate of the forest. In Bangladesh, higher annual carbon sequestration rates are noticed in plantation forests, while natural forests are 
maintaining higher long term capacities for carbon sequestration [12,13]. Forest vegetation plays a prominent role in carbon sequestration in terrestrial ecosystems, wherein the northern hemisphere, forest, maintains global carbon balance through critical carbon sequestration [14]. Trees are considered as the most crucial carbon sink and biomass source in the world, including various uses such as food, fuelwood, domestic, and other industrial uses. Due to extreme disturbances in climate, forest tree biomass is decreasing, and it is a matter of risk that if this occurrence is running as a continuous rate, the forest biomass will ruin that will release more carbon to the earth [15]. From 1990 to 2000, 9.3 million hectares of forest land was cleared per year for which in the meantime, about 465 million ton carbon released to the atmosphere [16]. Bangladesh was once densely forested, but this has been cleared for timber, converted to agriculture or cut for firewood where in recent times, the speed of deforestation has increased with forest cover shrinking from $18 \%$ in 1927 to a more $6 \%$ today [17]. It has been estimated that the tree tissues store $190 \mathrm{tC} / \mathrm{ha}$ in the forests of Bangladesh, indicating that per ha forests cover can demand remarkable payment for carbon sequestration and with this capital may be developed in the forestry sector in multipurpose aspects [13]. The presence of forests and trees provide benefit to the community in many ways, such as adapting to climate change impacts, maintaining natural resources, and improving sustainable development [18].

The participatory carbon measurement program is essential to reduce the transaction cost related to expert involvement in the field of carbon sequestration. A participatory carbon measurement practice in the indigenous communities in Indonesia's Kutai Barat and Mahakam Ulu districts was conducted to enhance the capability of local people in the field of estimating and monitoring the forests' carbon by sorting out the members of the community has long-established knowledge on forests including aims to develop technical capacities, accurate carbon inventories, forest conservation and empower local communities, etc. [19]. 
Many studies were conducted for measuring tree carbon [2, 10, 14, 16, 20-32], but the measurement of tree carbon in a participatory approach cannot be noticed in enough scale. Furthermore, it has been seen that no study was conducted on participatory tree carbon measurement in Bangladesh yet. From the literature review, it can be said that trees are the most critical carbon pool of forest [23, 25, 30-32]. So, the objectives of the research were to estimate the tree carbon in the Komolchari Village Common Forests (VCF) under Chittagong Hill Tracts (CHT) and to judge the efficacy and appropriateness of the participatory carbon measurement in terms of data collection. This study will enrich the knowledge pool of participatory carbon measurement in Bangladesh.

\section{Materials and Methods}

The study was conducted in the wet mixed forests in the Khagrachari Sadar Upazila of Khagrachari District under the CHT of Bangladesh. One VCF and the corresponding selected members were studied for observing the baseline carbon stock and building up the capacity of the local community members on carbon measurement. The VCF was selected purposively as a medium growth natural forest. Participatory delineation was the first-hand effort and human resource management, i.e., team formation, orientation (Theoretical and practical), detail training of the local community members was the second task of the research work. Participation in permanent plot marking, data collection by the local trained crews, and professional carbon inventory were the significant activities of the research work.

A total of 15 trainees were selected for the study purposively. With the discussion of the Karbary (leader of the social organization), poorer households were selected purposively. After selecting the participants and putting them under a training scheme, the behavior of the participants towards the VCF was independently monitored. 
Danesh Miah, Md. Arif Chowdhury and Mohammed Jashimuddin

\subsection{Description of the study area}

The study was conducted from March 2014 to August 2015 in Komolchari VCF (locally called reserve) in Buarchari Mouza (Mouza no- 264) under Khagrachari Sadar Upazila. The Komolchari VCF is of 128 hectares managed by the local ethnic community as a co-management agent. The VCF is about $5 \mathrm{~km}$ away from the village Komolchari.

A view reflecting the location of Komolchari VCF in Khagrachari district appears in figure1.

The VCF was primarily considered for three strata based on the density of the trees. The strata were dense, moderately dense, and spared. However, at the final data collection, we did not consider the different strata because, based on the anticipated three strata, there was no significant difference in tree density in the study area.

\subsection{Sampling and data collection}

From the VCF, a total of 57 permanent sample plots were studied. The plots were selected randomly using the superimposed grids on the VCF map through the local peoples' participation. The plots were circular in shape.

For measuring trees, plots of the $10 \mathrm{~m}$ radius were demarcated (Figure 2). From the same plot, a sub-plot of a $5 \mathrm{~m}$ radius for saplings was demarcated. For a convenient demarcation of the boundary of the plot, a nylon rope of $62.8 \mathrm{~m}$ length for $10 \mathrm{~m}$ radius and $31.4 \mathrm{~m}$ length for $5 \mathrm{~m}$-radius plot were used. For measuring the radius of the plots, $10 \mathrm{~m}$ and $5 \mathrm{~m}$ length nylon ropes were used. Abney level was used for measuring the slope of the plot. 


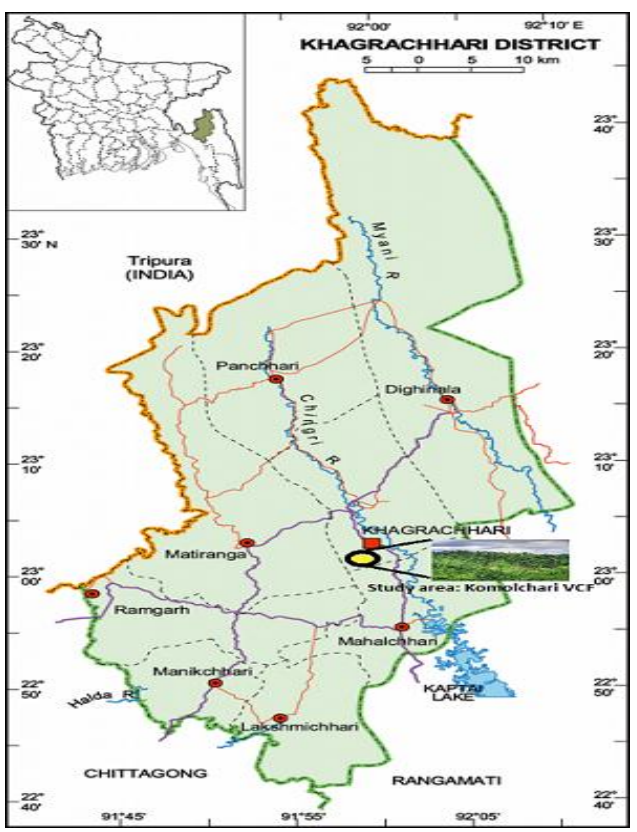

Figure 1: Location of Komolchari Village Common Forest in Khagrachari district under the Chittagong Hill Tracts.

The center of the plot was recorded as the global coordinates (latitude, longitude) assisted by the Global Positioning System (GPS). For further monitoring of the same plot, the coordinates of the center of the plots were put in the system of proximity alarm of the GPS. Plots were distributed between the latitudes ranging from $23^{\circ} 05.006^{\prime}$ to $23^{\circ} 05.988^{\prime}$ and longitudes from $92^{\circ} 01.774^{\prime}$ to $92^{\circ} 02.206^{\prime}$. 
8 Danesh Miah, Md. Arif Chowdhury and Mohammed Jashimuddin

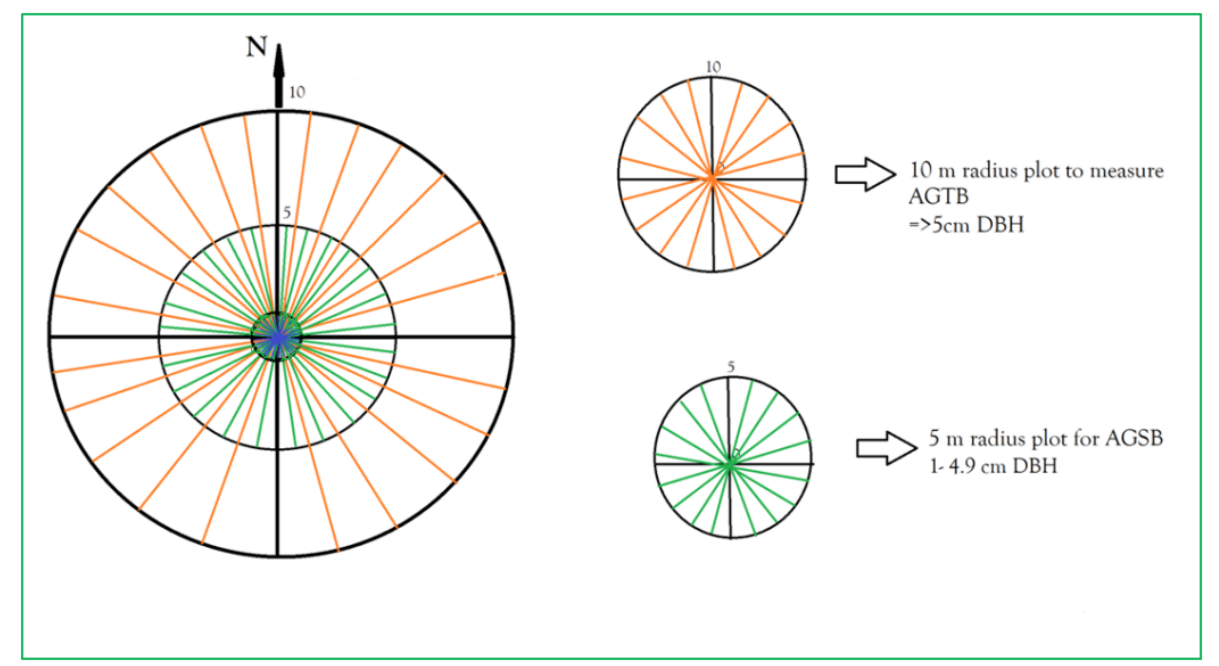

Notes: AGTB=Aboveground Tree Biomass; AGSB=Aboveground Sapling Biomass; DBH= Diameter at Breast Height

Figure 2: Demarcation of circular plots for sampling in the Komolchari Village Common Forests of Khagrachari under the Chittagong Hill Tracts.

The forestry professionals collected data on tree and sapling, and participants group (5 persons consisted of one group) also collected data following the same way. Professional records were enrolled by the experts from the Institute of Forestry and Environmental Sciences, Chittagong University (IFESCU), who had standard knowledge on forest inventory.

Bird-eye view of the study area indicating the waypoints of 57 permanent plots and whole covered area by connecting the border waypoints is presented in the following figure 3 . 


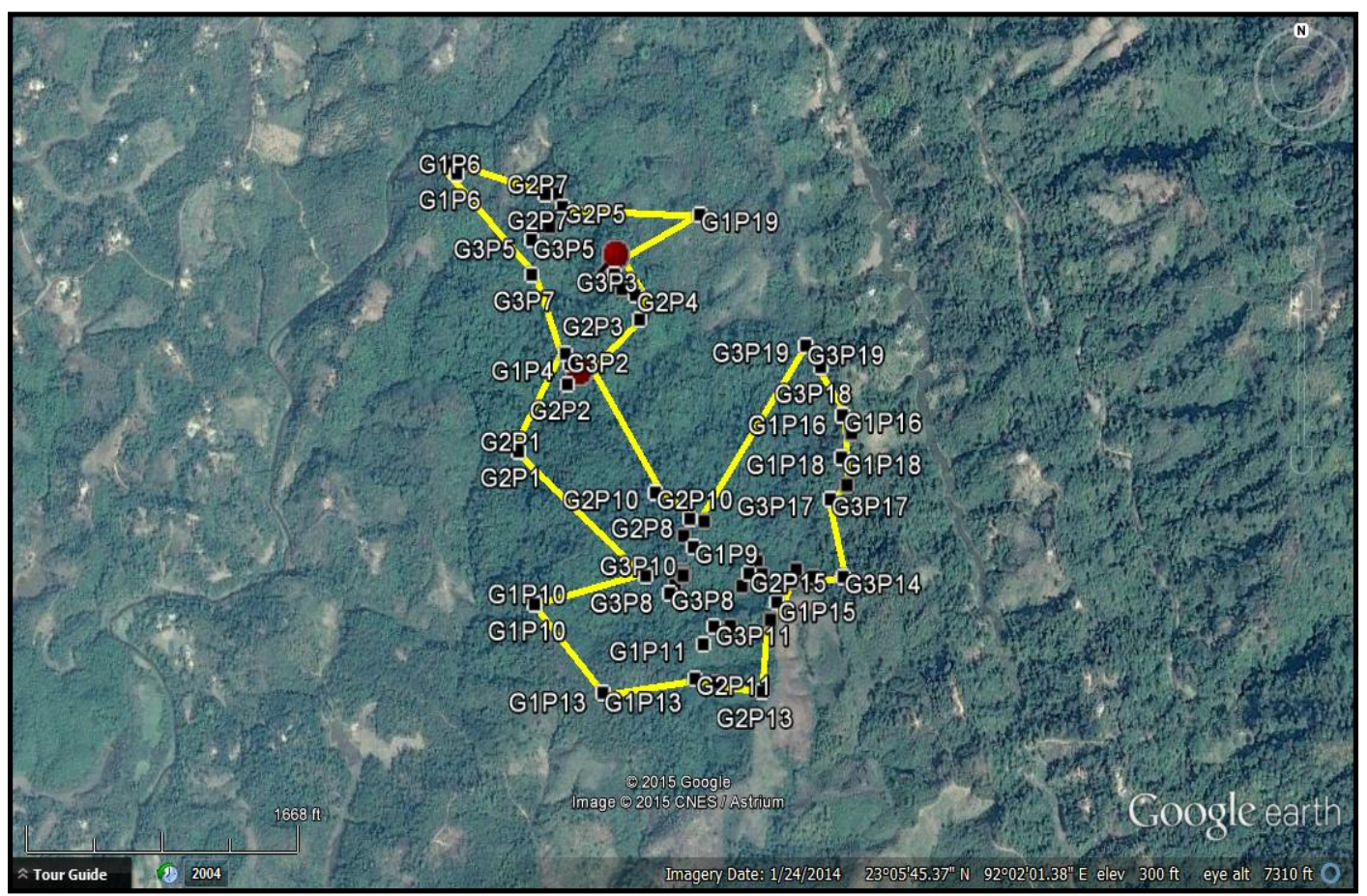

Figure 3:The demarcated study area connecting the boundary waypoints indicating all waypoints of permanent plots in Khagrachari under the Chittagong Hill Tracts.

\subsection{Collection of primary data}

A structured data record sheet was formed to record data on diameter at breast height (dbh) of tree and sapling. Diameter at breast height (dbh) ranged from 1 to $4.9 \mathrm{~cm}$ and more than $5 \mathrm{~cm}$ determined as sapling and tree, respectively. Slide calipers and measuring tapes were used to measure dbh of sapling and tree, respectively. Measurement on dbh of tree and sapling was conducted by the forestry professionals as well as local participants' groups for the same plot. 


\subsection{Data Analysis}

All data were input in the MS Excel to analyze, and the allometric regression equation was used for measuring the above-ground and below-ground biomass in the trees. The carbon was measured based on the biomass-carbon conversion factor.

All trees with dbh (diameter at breast height) of more than $5 \mathrm{~cm}$ were individually identified for the vegetation study. Diameter at breast height of the trees was collected to use in the allometric equation of the aboveground biomass. As speciesspecific allometric equations were not available for this study, a general equation only was used. The above-ground biomass density (ABD) of trees was estimated using the allometric model described by Pearson et al. [33]:

Biomass, $k g /$ tree $=\exp \left(-2.289+2.649 * \ln d b h-0.021 * \ln d b h^{2}\right)$, where $\ln$ is the natural logarithm.

The belowground biomass density (BBD) was found using the following allometric equation described by Pearson et al. [33]:

$B B D=\exp (-1.0587+0.8836 * \ln \mathrm{ABD})$ where $\ln$ is the natural logarithm.

The biomass was then converted to carbon stock using the factor 0.5 for estimating both aboveground carbon density (ACD) and belowground carbon density (BCD) [33].

\subsection{Data comparison}

After estimating the available tree carbon by using data collected by the local participants' group and the forestry professionals, a comparison by the ' $t$ ' test among the results was conducted. Results of the t-test are based on two-sided tests assuming equal variances with significance level. Tests were adjusted for all pair-wise comparisons within a row of each innermost sub-table using the Bonferroni correction. 


\section{Results and Discussion}

\subsection{General information about the richness of trees and saplings}

Diameter at breast height (dbh) of 2755 trees was recorded. It was estimated that mean $\mathrm{dbh}(\mathrm{cm})$ in the study area was $9.32 \pm 0.09$. Here, the mode of the dbh of the tree was $6 \mathrm{~cm}$, and the median was $7.67 \mathrm{~cm}$, with the ranges from 5 to $46 \mathrm{~cm}$. It was found that about $76 \%$ of trees had a dbh between 5 to $9.9 \mathrm{~cm}$, which was the dominant one comparing the others. On the other hand, few numbers of trees were found, having a dbh range of $25+\mathrm{cm}$ (about $2 \%$ ). Furthermore, almost $18 \%, 6 \%$, and $3 \%$ of trees contained the dbh from 10 to $14.9 \mathrm{~cm}, 15$ to $19.9 \mathrm{~cm}$, and 20 to $24.9 \mathrm{~cm}$, respectively.

Diameter at breast height (dbh) of 5285 saplings was recorded. It was estimated that the mean dbh $(\mathrm{cm})$ of the saplings in the study area was $2.47 \pm 0.02$. Here, the mode of the dbh was $1.2 \mathrm{~cm}$, and the median was $2.30 \mathrm{~cm}$, with the ranges from 1 to 4.9 $\mathrm{cm}$. It was found that Macher chokh bichi (Bridelia sp.) had the highest percentage distribution among all the species. Furthermore, Sanisil (Mitragyna sp.) and Kanak (Schima wallichi) were the second and third stages in the case of percentage distribution among all the sapling species.

Among the tree and sapling species in the study area, Amilik (Emblica officinalis) was the most dominant species in number (1475) where Burogach (Macaranga denticulata) was the second most dominant species in number (464) where Macher chokh Bichi (Bridelia sp.) (445) was the third most dominant. Suguijja pata (Litsea $s p$.), Mon buro (Pterospermum acerifolium), Khonagula (Oroxylum indicum), Fulkumari (Hymenodictyon orixensis), Chickrashi (Chikrassia tabularis) were less in number (1) among all the recorded dbh of species. Furthermore, Asar (Grewia nervosa), Hoja (Celastrus robustus), Jam (Syzygiam sp.), Jata Boroi (Anogeisus acuminata), Kanak (Schima wallichi), Moner moto gach (Actinodephne angustifolia), Narissa (Trema orientalis), Nuinna bichi (Macranga indica) and 
Sanisil (Mitragyna sp.) were foundas 114, 103, 125, 117, 160, 129, 150, 112 and 213 in number, respectively.

A total of 74 species of trees were identified in the study area. In Idgaon Reserve of the Cox's Bazar North forest division, 66 tree species were recorded [34]. In the degraded natural forest land of the Chittagong South Forest Division, a total of 21 trees species were recorded [35]. In a study in Chunati Wildlife Sanctuary of Chittagong, 99 tree species were recorded [36]. So it can be said that the tree species richness in the Komolchari VCF is quite high.

\subsection{Biomass in trees}

The aboveground biomass density in trees was $123.777 \pm 26.746$ tha $^{-1}$ and belowground biomass density was $23.620 \pm 4.511 \mathrm{tha}^{-1}$, and the total biomass density was $147.40 \pm 31.26$ tha $^{-1}$ from the data collected by forestry experts group (Table 1). Again, in the measurement of local participants, it shows that aboveground biomass density, average belowground biomass density, and average total biomass density in trees was $113.95 \pm 23.51$ tha $^{-1}, 22.01 \pm 4.03$ tha $^{-1}$ and $135.95 \pm 27.54$ tha $^{-1}$, respectively.

Table 1. Biomass of trees in Komolchari Village Common Forest in Khagrachari under the Chittagong Hill Tracts.

\begin{tabular}{|c|c|c|c|}
\hline \multirow[t]{2}{*}{ Parameters } & & \multicolumn{2}{|c|}{ Measuring group } \\
\hline & & $\begin{array}{l}\text { Forestry experts } \\
\left(\text { tha }^{-1}\right)\end{array}$ & $\begin{array}{l}\text { Local participants } \\
\left(\text { tha }^{-1}\right)\end{array}$ \\
\hline $\begin{array}{l}\text { Aboveground } \\
\text { density }\end{array}$ & biomass & $123.78 \pm 26.75^{*}$ & $113.95 \pm 23.51$ \\
\hline $\begin{array}{l}\text { Belowground } \\
\text { density }\end{array}$ & biomass & $23.62 \pm 4.51$ & $22.01 \pm 4.03$ \\
\hline Total biomass density & & $147.40 \pm 31.26$ & $135.95 \pm 27.54$ \\
\hline
\end{tabular}


In figure 4 , the horizontal straight line in the boxes represents the total biomass density of trees, which was obtained from forestry experts and local participants as well. The line for both of the groups remains inside the box, indicating the measurements by forestry experts and local participants are almost the same.

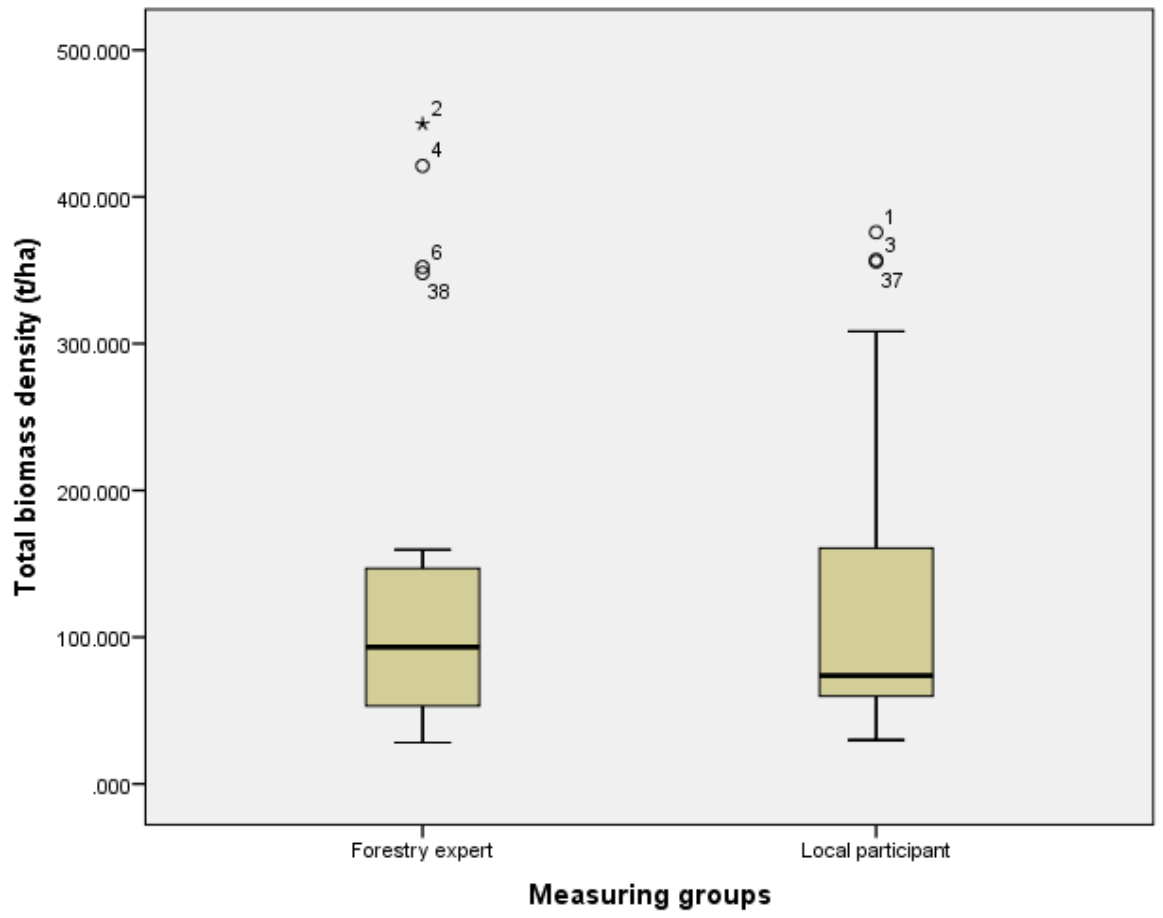

Figure 4: Data distribution of the total biomass density $\left(\right.$ tha $\left.^{-1}\right)$ of trees in the Komolchari Village Common Forest in Khagrachari under the Chittagong Hill Tracts.

The representing line of total biomass density data in the graph over forestry experts and local participants groups shows no significant dispersion evident by the paired ttest. The results from the study revealed that the aboveground biomass density of trees from the measurement of forestry experts and local participants were $123.78 \pm 26.75$ tha $^{-1}$ and $113.95 \pm 23.51$ tha $^{-1}$, respectively. On the other hand, 
14 Danesh Miah, Md. Arif Chowdhury and Mohammed Jashimuddin

belowground biomass density of trees for forestry experts and local participants were $23.62 \pm 4.51 \mathrm{tha}^{-1}$ and $22.01 \pm 4.03 \mathrm{tha}^{-1}$, respectively (Table 1). Shin et al. [37] found that the aboveground biomass of the 13 tree species of ages from 6 to 23 years were $155.50 \pm 9.08$ tha $^{-1}$ and $23.32 \pm 1.36$ tha $^{-1}$, respectively, in the plantation forests in Chittagong areas of Bangladesh. The aboveground biomass of the present study is lower than the study of Shin et al. [37]. The present study considers the naturally grown trees in theKomolchari VCF in the Chittagong Hill Tracts. The species found in the present VCF are not fast-growing as the species found in the plantation of the Chittagong area [37-39]. For this reason, the aboveground biomass is lower in quantity than that of the plantation forests in Chittagong. A study in the USA shows that in the softwood forest, the range of aboveground biomass was 2.5-10 tha ${ }^{-1}$ [21], and another study in India depicts that the aboveground and belowground biomass for 95 farmlands was $0.02 \pm 0.01$ tha $^{-1}$ and 0.01 tha $^{-1}$ [40]. Again in the case of total biomass density from the measurement of forestry experts and local participants were $147.40 \pm 31.26$ tha $^{-1}$ and $135.95 \pm 27.54$ tha $^{-1}$, respectively, in the Komolchari VCF. From a study, it was found that the range of biomass in Beijing was from 1.90 tha $^{-1}$ to 54.74 tha $^{-1}$ (Yu et al. 2011b). From another study of all closed forests in Asia, the average biomass was 328 tha $^{-1}$ while ranging from 160-538 tha ${ }^{-1}$ and for all open forest was 80 tha $^{-1}$ while ranging 40-140 tha ${ }^{-1}$ [41]. From this discussion, it can be said that the concentration (tha ${ }^{-1}$ ) of aboveground biomass density, belowground biomass density, and total biomass density of Komolchari VCF present theright stock level. Baten et al. [38] confirmed that the VCF in Rangamati and Bandarban district is more affluent in the biodiversity of tree species than that of the government forest. They also affirmed that the traditional knowledge of the ethnic people tends to maintain the balance between forest produce extraction and its conservation. The crucial role of the VCF to fulfill the demands of the forest produces of the ethnic communities in the Chittagong Hill Tracts of Bangladesh is also articulated by Jashimuddin and Inoue [39]. Indigenous knowledge towards sustainable forest management is the critical factor to the better tree species diversity in the VCF is 
evident by the studies of Baten et al. [38], Jashimuddin and Inoue [39] and Islam et al. [42].

\subsection{Biomass in sapling}

The average aboveground, belowground, and total biomass density in saplings were $27.26 \pm 2.90$ tha $^{-1}, 6.37 \pm 0.60$ tha $^{-1}$, and $33.63 \pm 3.50$, respectively, from the data collected by the forestry experts group (Table 2). Again, the measurement of local participants shows that average aboveground, belowground, and total biomass density in saplings were $26.24 \pm 2.562$ tha $^{-1}, 6.170 \pm .53$ tha $^{-1}$ and $32.41 \pm 3.09$ tha $^{-1}$, respectively.

Table 2. Biomass of saplings in Komolchari Village Common Forest in Khagrachari under the Chittagong Hill Tracts.

\begin{tabular}{lll}
\hline Parameters & \multicolumn{2}{c}{ Measuring group } \\
\cline { 2 - 3 } & $\begin{array}{l}\text { Forestry experts } \\
\left(\text { tha }^{-1}\right)\end{array}$ & $\begin{array}{l}\text { Local participants } \\
\left(\text { tha }^{-1}\right)\end{array}$ \\
\hline Aboveground biomass density & $27.26 \pm 2.90^{*}$ & $26.24 \pm 2.56$ \\
Belowground biomass density & $6.37 \pm 0.60$ & $6.17 \pm .53$ \\
Total biomass density & $33.63 \pm 3.50$ & $32.41 \pm 3.09$ \\
\hline \multicolumn{2}{c}{ *Standard Error of Mean } &
\end{tabular}

In figure5, the horizontal straight line in the boxes represents the total biomass density of saplings, which was obtained from forestry experts and local participants as well. The line for both of the groups remains inside the box, indicating the measurements by forestry experts and local participants are almost the same.

The representing line of total biomass density data in the graph over forestry experts and local participants groups shows no significant dispersion evident by the paired ttest. 
16 Danesh Miah, Md. Arif Chowdhury and Mohammed Jashimuddin

The total estimated biomass density of the Komolchari VCF from a measurement of forestry experts and local participants was $33.631 \pm 3.497$ tha $^{-1}$ and 32.410 \pm 3.094 tha ${ }^{-1}$, respectively. In a study in Beijing shows that the average biomass in forest vegetation of Beijing is 26.66 tha $^{-1}$ [14]. It can be said that from the above-given study, Komolchari VCF contains an excellent density of biomass.

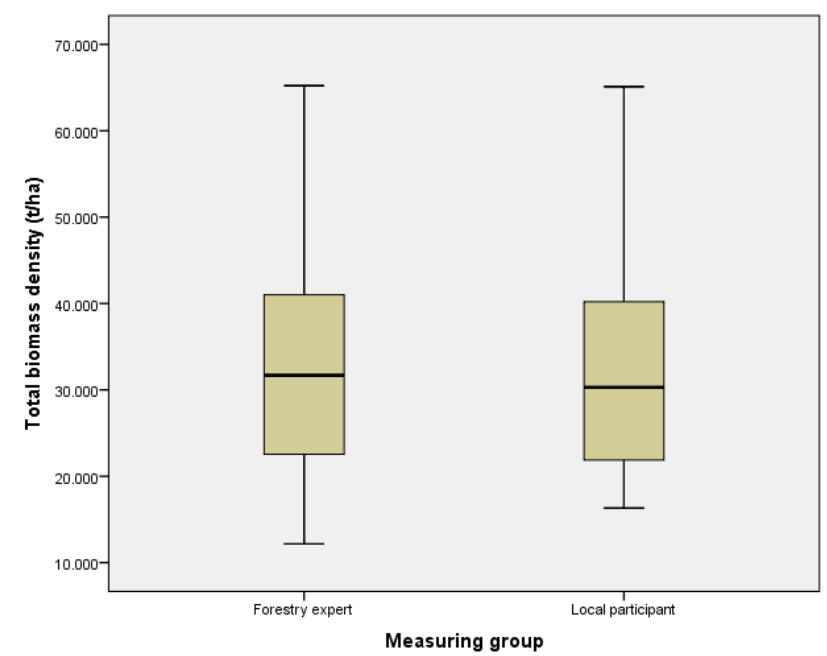

Figure 5: Data distribution of the total biomass density (tha ${ }^{-1}$ ) of saplings in the Komolchari Village Common Forest in Khagrachariunder the Chittagong Hill Tracts.

\subsection{Carbon in trees}

The study found that the aboveground, belowground, and total carbon density in trees were $61.89 \pm 13.37$ tha $^{-1}, 11.81 \pm 2.26$ tha $^{-1}$, and $73.70 \pm 15.63$ tha $^{-1}$, respectively, from the data collected by forestry experts (Table 3 ). On the other hand, the results from the data collected by the local participants showed that the aboveground, belowground, and total carbon density in trees were $56.97 \pm 11.76$ tha $^{-1}, 11.01 \pm 2.01$ tha ${ }^{-1}$ and $67.98 \pm 13.77$ tha $^{-1}$, respectively. There was no significant $(p<0.05)$ difference between the carbon amount measured by the forestry experts and the local participants. 
Table 3. Carbon of trees in the Komolchari Village Common Forest in Khagrachari under the Chittagong Hill Tracts.

\begin{tabular}{|c|c|c|c|}
\hline \multirow[t]{2}{*}{ Parameters } & & \multicolumn{2}{|c|}{ Measuring group } \\
\hline & & $\begin{array}{l}\text { Forestry experts } \\
\left(\text { tha }^{-1}\right)\end{array}$ & $\begin{array}{l}\text { Local participants } \\
\left(\text { tha }^{-1}\right)\end{array}$ \\
\hline $\begin{array}{l}\text { Aboveground } \\
\text { density }\end{array}$ & carbon & $61.89 \pm 13.37 *$ & $56.97 \pm 11.76$ \\
\hline $\begin{array}{l}\text { Belowground } \\
\text { density }\end{array}$ & carbon & $11.81 \pm 2.26$ & $11.01 \pm 2.01$ \\
\hline Total carbon density & & $73.70 \pm 15.63$ & $67.98 \pm 13.77$ \\
\hline
\end{tabular}

In figure 6, the horizontal straight line in the boxes represents the total carbon density of trees which was obtained from forestry experts and local participants as

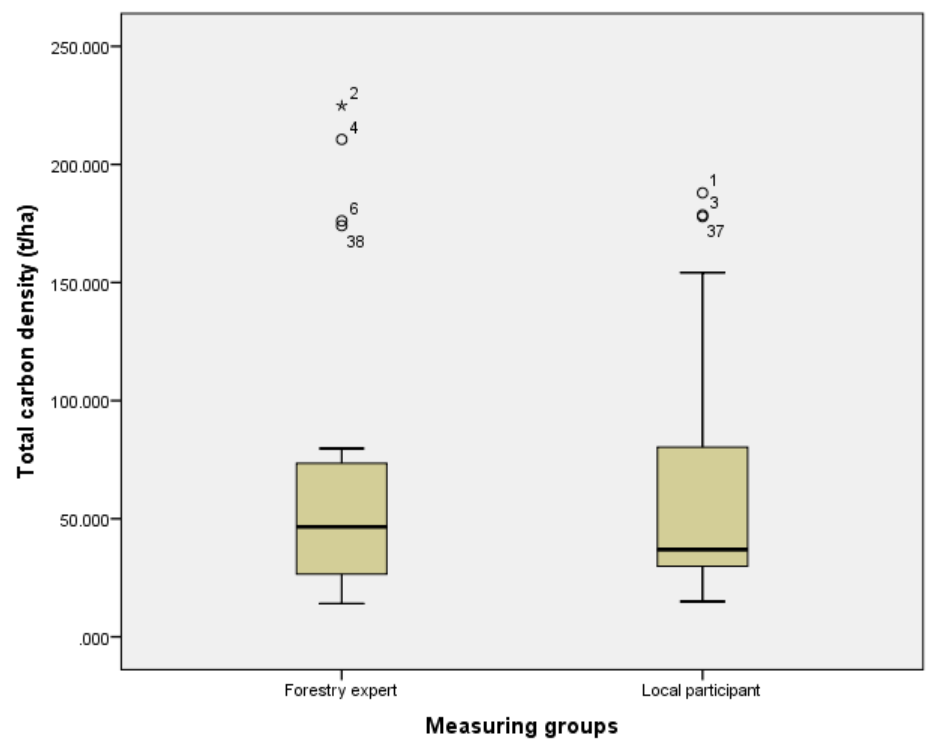

Figure 6: Data distribution of the total carbon density $\left(\right.$ tha $\left.^{-1}\right)$ of trees in the Komolchari Village Common Forest in Khagrachari under the Chittagong Hill Tracts. 
well. The line for both of the groups remains inside the box indicating the measurements by forestry experts and local participants are almost the same.

The representing line of total carbon density in the graph over forestry experts and local participants groups shows no significant dispersion. It has also been evident by the paired t-test.

It is revealed from the study that the total carbon density of trees for forestry experts and local participants were 73.699 \pm 15.626 tha $^{-1}$ and $67.975 \pm 13.768$ tha $^{-1}$, respectively. From a study, it is found that the forests of Bangladesh can sequestrate 92 tha $^{-1}$ on average [13]. Furthermore, in another study in Kashmir Himalaya, India shows that the tree carbon stocks ranged from 45.4 to 135.6 tha $^{-1}$ [43]. Again in a study in India depicts that, carbon density for managed forests was 42 tha $^{-1}$ [23]. The analysis indicates that the amount of total carbon in trees in Komolchari VCF shows a higher growing stock in comparison to the other available studies.

\subsection{Carbon in saplings}

The average aboveground, belowground, and total carbon density in saplings of Komolchari VCF were $13.63 \pm 1.45$ tha $^{-1}, 3.19 \pm 0.30$ tha $^{-1}$, and $16.82 \pm 1.75$ tha $^{-1}$, respectively, from the data collected by forestry experts (Table 4). Those were $13.12 \pm 1.28$ tha $^{-1}, 3.09 \pm 0.27$ tha $^{-1}$ and $16.21 \pm 1.55$ tha $^{-1}$, from the data collected by local participants. There was no significant difference between the carbon amount measured by the forestry experts and the local participants.

In figure 7 , the horizontal straight line in the boxes represents the total carbon density of saplings, which was obtained from forestry experts and local participants as well. The line for both of the groups remains inside the box indicating the measurements by forestry experts and local participants are almost the same. 
Table 4. Carbon of saplings in the Komolchari Village Common Forest in Khagrachari under Chittagong Hill Tracts.

\begin{tabular}{lll}
\hline Parameters & \multicolumn{2}{c}{ Measuring group } \\
\cline { 2 - 3 } & $\begin{array}{l}\text { Forestry experts } \\
\left(\text { tha }^{-1}\right)\end{array}$ & $\begin{array}{l}\text { Local participants } \\
\left(\text { tha }^{-1}\right)\end{array}$ \\
\hline Aboveground carbon density & $13.63 \pm 1.45^{*}$ & $13.12 \pm 1.28$ \\
Belowground carbon density & $3.19 \pm 0.30$ & $3.09 \pm 0.27$ \\
Total carbon density & $16.82 \pm 1.75$ & $16.21 \pm 1.56$ \\
\hline \multicolumn{2}{c}{ *Standard Error of Mean } &
\end{tabular}

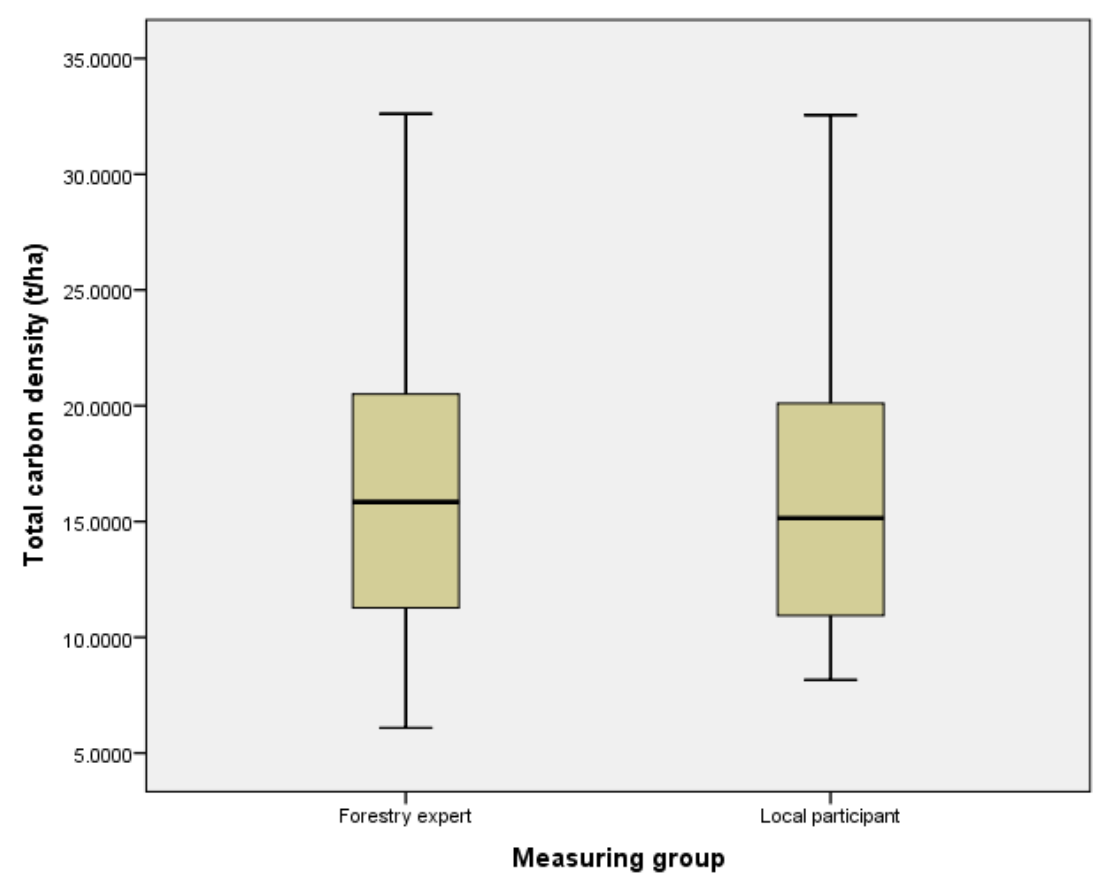

Figure 7: Data distribution of the total carbon density $\left(\right.$ tha $\left.^{-1}\right)$ of saplings in the Komolchari Village Common Forest in Khagrachari under the Chittagong Hill Tracts. 
The representing line of total carbon density of saplings in the graph over forestry experts and local participants groups shows no significant dispersion. It has also been evident by the paired t-test.

From the study, it was revealed that the total carbon density of saplings measured by the forestry experts and the local participants were $16.82 \pm 1.75$ tha $^{-1}$ and $16.21 \pm 1.55$ tha $^{-1}$, respectively. A study conducted in Beijing shows that the average carbon sequestration rate is 3.94 tha $^{-1}$ [14]. So, it can be said thatthe total carbon density of saplings in the Komolchari VCF is presented at a reasonable level.

\section{Conclusion}

The study confirms that the tree carbon measurement through a participatory approach in Komolchari village common forest was successfully conducted. The findings of the study highlight the mechanism to reduce the transaction cost to collect the data on tree carbon measurement. The estimated value of total biomass density of trees measured by the forestry experts and the local participants was 147.40 tha $^{-1}$ and 135.95 tha $^{-1}$, respectively, where total carbon density for trees measured by the forestry experts and the local participants were 73.70 tha $^{-1}$ and 67.98 tha $^{-1}$, respectively. Furthermore, in the case of saplings, the estimated total biomass density measured by the forestry experts and the local participants was 33.63 tha $^{-1}$ and $32.41 \mathrm{tha}^{-1}$, respectively, where estimated total carbon density measured by the forestry experts and the local participants were 16.82 tha $^{-1}$ and 16.21 tha $^{-1}$, respectively. All of the findings revealed that Komolchari village common forest had a good stock of tree biomass and carbon. It will be cheaper to estimate the carbon through a participatory approach, which has a potential scope to maintain a carbon market through REDD+. This first attempt of participatory tree carbon measurement in the natural forests in Bangladesh will enrich the knowledge pool of tree carbon measurement. It will complement the act of National Forest Monitoring System (NFMS) and Measurement, Reporting and Verification (MRV) of the REDD+ requirements in Bangladesh. 


\section{Acknowledgment}

The study was conducted under a project titled "Participatory carbon measurement for REDD+" funded by Arannayk Foundation, Dhaka, Bangladesh.

\section{References}

[1] F. Seymour and J. Busch: "Why forests? Why now?: The science, economics, and politics of tropical forests and climate change", 2016. Brookings Institution Press.

[2] B. P. Sharma, P. Shyamsundar, M. Nepal, S. Pattanayak and B. S. Karky: "Can carbon be sequestered in community forests? Learning how to fulfill a global agenda through local institutions in Nepal", 2015. SANDEE (The South Asian Network for Development and Envrionmental Economics), Kathmandu, Nepal, p. 2.

[3] E. Merger, C. Held, T. Tennigkeit, and T. Blomley: Carbon Balance and Management, 2012, 7(1), 9.

[4] S. K. Entenmann, T. A. Kaphegyi and C. B. Schmitt: Environmental Management, 2014, 53 (2), 300-317.

[5] P. Dargusch, K. Lawrence, J. Herbohn, and Medrilzam: Small-Scale Forestry, 2010, 9(4), 485-499.

[6] K. Massarella, S. M. Sallu, J. E. Ensor, and R. Marchant: World Development, 2018, 109, 375-385.

[7] S. Movik, G. Birikorang, A. Enright, G. Kajembe, L. Lima, S. Marostica, T. M. Pinto, G. Nabanoga, M. Nantongo and J. Namaalwa: "Socio-economic conditions in REDD+ pilot areas: A synthesis of five baseline surveys", 2012. International Institute for Environment and Development (IIED), London, UK, p. 46.

[8] B. Subedi, S. Pandey, A. Pandey, E. Rana, S. Bhattarai, T. Banskota, S.

Charmakar and R. Tamrakar: "Guidelines for measuring carbon stocks in community managed forests", 2011. Asia Network for Sustainable Agriculture and Bioresources (ANSAB); Federation of Community Forest Users, Nepal (FECOFUN); International Centre for Integrated Mountain Development (ICIMOD), Kathmandu, Nepal, p. 76.

[9] UN-REDD: Bangladesh National Programme: Program brief, 2019. Vol 2019, UNREDD Bangladesh National Programme, Dhaka. 
[10] H. K. Gibbs and S. Brown: "Geographical distribution of biomass carbon in tropical southeast Asian forests: an updated database for 2000", 2008. Oak Ridge National Laboratory, Tennessee, USA, p. 66.

[11] J. Rotter and K. Danish: Journal of Forestry, 2000, 98 (5), 38-47.

[12] S. Bass, O. Dubois, P. Moura Costa, M. Pinard, R. Tipper and C. Wilson: "Rural livelihoods and carbon management", 1997. International Institute for Environment and Development (IIED),, London, UK, p. 94.

[13] M. D. Miah, M. Y. Shin and M. Koike: "Forests to climate change mitigation: Clean Development Mechanism in Bangladesh", 2011. Springer, Heidelberg.

[14] Y. Xiao, K. An, G. Xie, C. Lu and B. Zhang: Chinese Geographical Science, 2011, 21(3), 279-289.

[15] S. G. Rolim, R. M. Jesus, H. E. Nascimento, H. T. Do Couto and J. Q. Chambers: Oecologia, 2005, 142(2), 238-246.

[16] N. K. Phat, W. Knorr and S. Kim: Forest Ecology and Management, 2004, 191(1-3), 283-299.

[17] FAO: National Forest and Tree Resources Assessment 2005-2007, Bangladesh, 2017. Food and Agriculture organization (FAO), Dhaka.

[18] J. S. Pender: Church of Bangladesh Social Development Programme, Dhaka, 2008.

[19] WWF: "Empowering communities through participatory carbon measurement in Indonesia", 2014. WWF Forest and Climate Programme, Gland, Switzerland, p. 6.

[20] S. Brown: "Estimating biomass and biomass change of tropical forests: a primer", 1997. Food \& Agriculture Org.

[21] S. Brown: Environmental Pollution, 2002, 116(3), 363-372.

[22] J. Frouz, V. Pižl, E. Cienciala, and J. Kalčík: Biogeochemistry, 2009, 94(2), 111 121.

[23] C. A. Hall and J. Uhlig: Canadian Journal of Forest Research, 1991, 21(1), 118131.

[24] S. P. Hamburg: Mitigation and Adaptation Strategies for Global Change, 2000, 5(1), 25-37.

[25] G. Haripriya: Biomass and Bioenergy, 2000, 19(4), 245-258.

[26] M. L. Kaonga and T. P. Bayliss-Smith: Agroforestry Systems, 2009, 76(1), 37-51.

[27] R. Kongsager, J. Napier and O. Mertz: Mitigation and Adaptation Strategies for Global Change, 2013, 18(8), 1197-1213. 

Hill Tracts

[28] M. Lal and R. Singh: Environmental Monitoring and Assessment, 2000, 60(3), 315327.

[29] R. Lasco and M. Cardinoza: Mitigation and Adaptation Strategies for Global Change, 2007, 12(2), 243-257.

[30] B. M. Shrestha and B. R. Singh: Nutrient cycling in Agroecosystems, 2008, 81(2), 179-191.

[31] Q. Chen, W. Xu, S. Li, S. Fu and J. Yan: Chinese Science Bulletin, 2013, 58(13), 1551-1557.

[32] Y. Liu, G. Yu, Q. Wang, Y. Zhang and Z. Xu: Science China Life Sciences, 2014, 57(12), 1218-1229.

[33] T. Pearson, S. Walker and S. Brown: "Sourcebook for land use, land-use change and forestry projects", 2005. Winrock International.

[34] M. K. Hossain: Journal of Forestry and Environment, 2001, 1(1), 102-110.

[35] M. D. Miah and S. S. Rahman: The Chittagong University Journal of Science, 2014, 36, 115-135.

[36] A. Mamun, M. A. Hossain, M. Hossain, and S. Alam: Indian Forester, 2015, 141 (5), 566-572.

[37] M. Y. Shin, M. D. Miah and K. H. Lee: Journal of Environmental Management, 2007, 82(2), 260-276.

[38] M. A. Baten, N. A. Khan, R. Ahammad, and K. Misbahuzzaman: "Village common forests in Chittagong Hill Tracts, Bangladesh: balance between conservation and exploitation", 2010. Dhaka.

[39] M. Jashimuddin and M. Inoue: Open Journal of Forestry, 2012, 2, 121-137.

[40] P. Sudha, V. Ramprasad, M. Nagendra, H. Kulkarni and N. Ravindranath: Mitigation and Adaptation Strategies for Global Change, 2007, 12(6), 1131-1152.

[41] S. Brown, A. J. Gillespie and A. E. Lugo: Forest Science, 1989, 35(4), 881-902.

[42] K. Islam, M. Jashimuddin and N. Hossain: Environment, Earth and Ecology, 2017, 1(2), 39-51.

[43] J. A. Dar and S. Sundarapandian: Environmental Monitoring and Assessment, 2015, 187(2), 55. 